REVISTA DE LA

UNIÓN MATEMÁTICA ARGENTINA

Vol. 59, No. 2, 2018, Pages 241-254

Published online: December 6, 2017

\title{
CYCLIC GROUPS WITH THE SAME HODGE SERIES
}

\author{
DARYL R. DEFORD AND PETER G. DOYLE
}

\begin{abstract}
The Hodge series of a finite matrix group is the generating function for invariant exterior forms of specified order $p$ and degree $k$. Lauret, Miatello, and Rossetti gave examples of pairs of non-conjugate cyclic groups having the same Hodge series; the corresponding space forms are isospectral for the Laplacian on $p$-forms for all $p$, but not for all natural operators. Here we explain, simplify, and extend their investigations.
\end{abstract}

\section{TERMinOLOGY AND NOTATION}

We adopt terminology and notation to avoid some common headaches.

'Just if'. We follow John Conway in using 'just if' in place of the more cumbersome 'if and only if'.

Modular arithmetic. $a \equiv_{q} b$ means $a$ is equivalent to $b \bmod q$. We write $\mathbf{Z}_{q}$ for $\mathbf{Z} / q \mathbf{Z}$, and $\mathbf{Z}_{q}^{\star}$ for its invertible elements, taking $\mathbf{Z}_{1}^{\star}=\{0\}$. For $a \in \mathbf{Z}_{q}, b \in \mathbf{Z}_{q}^{\star}$ we write $a / q b$ for the quotient $\bmod q$.

Angles; roots of unity. We use $\tau=2 \pi$ in representing angles, because as $\mathrm{Vi}$ Hart [5] has so persuasively argued, $\pi$ is wrong. We write

$$
\omega_{q}=\exp (i \tau / q)
$$

for the standard $q$ th root of unity, so that

$$
\omega_{q}^{k}=\exp (i \tau k / q)=e^{i \tau \frac{k}{q}} .
$$

Unitary and orthogonal groups; conjugacy. As usual we write $U_{n} \subset$ $G L_{n}(\mathbf{C})$ for the $n$-by- $n$ unitary matrices, and $O_{n}=U_{n} \cap G L_{n}(\mathbf{R})$ for the orthogonal matrices.

When we say that two matrices or groups are 'conjugate', we mean that they are conjugate within $G L_{n}(\mathbf{C})$, so that the conjugating matrix can be any invertible complex matrix. Allowing this generality for the conjugating matrix is no big deal, because unitary matrices or groups that are conjugate within $G L_{n}(\mathbf{C})$ are already conjugate within $U_{n}$; real matrices or groups that are conjugate within

2010 Mathematics Subject Classification. 58J53.

The authors hereby waive all copyright and related or neighboring rights to this work, and dedicate it to the public domain. 
$G L_{n}(\mathbf{R})$ are already conjugate within $G L_{n}(\mathbf{R})$; orthogonal matrices or groups that are conjugate within $G L_{n}(\mathbf{C})$ are already conjugate within $O_{n}$.

We will be dealing with finite groups of matrices, which we will be interested in only up to conjugacy. Any finite group of complex matrices is conjugate to a subgroup of $U_{n}$; any finite group of real matrices is conjugate to a subgroup of $O_{n}$. So we may take our groups to be unitary - and if real, orthogonal - without sacrificing generality.

\section{HODGE SERIES}

Let $G \subset U_{n}$ be a finite group of $n$-by- $n$ complex matrices, assumed to be unitary. Any $g \in G$ is diagonalizable, with the roots $\lambda_{1}, \ldots, \lambda_{n}$ of its characteristic polynomial

$$
\chi_{g}(x)=\operatorname{det}\left(x I_{n}-g\right)=\prod_{i}\left(x-\lambda_{i}\right)
$$

being roots of unity.

Define the Hodge series

$$
\begin{aligned}
\Lambda_{G}(x, y) & =\frac{1}{|G|} \sum_{g} \frac{\operatorname{det}\left(I_{n}+y g\right)}{\operatorname{det}\left(I_{n}-x g\right)} \\
& =\frac{1}{|G|} \sum_{g} \frac{(-y)^{n} \chi_{g}(-1 / y)}{x^{n} \chi_{g}(1 / x)} .
\end{aligned}
$$

This series is a particular kind of Molien series: Crass [1, p. 31] calls it the 'exterior Molien series'. By a generalization of Molien's theorem (cf. Molien [10], Stanley [12]) this is the generating function for $G$-invariant exterior forms:

$$
\Lambda_{G}(x, y)=\sum_{p, k} P_{k}^{p} x^{k} y^{p},
$$

where $P_{k}^{p}$ is the dimension of the space of $G$-invariant $p$-forms whose coefficients are homogeneous polynomials of degree $k$ in $x_{1}, \ldots, x_{n}$.

For example we have

$$
\Lambda_{\left\{I_{n}\right\}}=\frac{(1+y)^{n}}{(1-x)^{n}}
$$

and

$$
\begin{aligned}
\Lambda_{\left\{ \pm I_{n}\right\}} & =\frac{1}{2}\left(\frac{(1+y)^{n}}{(1-x)^{n}}+\frac{(1-y)^{n}}{(1+x)^{n}}\right) \\
& =\frac{\frac{1}{2}\left(\left(1+x^{n}\right)\left(1+y^{n}\right)+\left(1-x^{n}\right)\left(1-y^{n}\right)\right)}{\left(1-x^{2}\right)^{n}} .
\end{aligned}
$$

Aside. Here's a more interesting example. The group $G_{120}$ of proper and improper symmetries of the icosahedron in Euclidean 3-space has Hodge series

$$
\Lambda_{G_{120}}=\frac{(1+x y)\left(1+x^{5} y\right)\left(1+x^{9} y\right)}{\left(1-x^{2}\right)\left(1-x^{6}\right)\left(1-x^{10}\right)} .
$$


As this might suggest, the algebra of invariant forms is generated by polynomial invariants of degrees 2,6,10 and their exterior derivatives of degrees 1,5,9. For the index-2 subgroup $G_{60}$ of proper symmetries we have

$$
\Lambda_{G_{60}}=\frac{\left(1+x^{15}\right)\left(1+y^{3}\right)+\left(x+x^{5}+x^{6}+x^{9}+x^{10}+x^{14}\right)\left(y+y^{2}\right)}{\left(1-x^{2}\right)\left(1-x^{6}\right)\left(1-x^{10}\right)} .
$$

This is a little harder to decipher, though the generating function for invariant polynomials, obtained by setting $y=0$, is clear enough:

$$
\Lambda_{G_{60}}(x, 0)=\frac{1+x^{15}}{\left(1-x^{2}\right)\left(1-x^{6}\right)\left(1-x^{10}\right)} .
$$

Here we see the $G_{120}$-invariants of degrees $2,6,10$, together with a new invariant of degree 15 (the product of the linear forms determining the 15 planes of symmetry of the icosahedron) whose square is $G_{120}$-invariant, though it itself is only $G_{60^{-}}$ invariant.

Exercise 1. Compute these two Hodge series.

Hint. Resist the temptation to consult Klein [8] or Doyle and McMullen [2]: You do not need to know the matrix groups explicitly, because the contribution of a matrix to the Hodge series depends only on its conjugacy class. This fact is the basis of the notion of 'almost-conjugacy' of groups, which we'll get to in a jiffy.

\section{Hodge EqUivalence}

We are interested in pairs of groups $G, H \subset U_{n}$ (and in particular, pairs of real groups $G, H \subset O_{n}$ ) having the same Hodge series, meaning that they have the same dimensions of spaces of invariant forms. We call such pairs Hodge-equivalent, and write $G \equiv_{\Lambda} H$.

Of course if the groups $G$ and $H$ are conjugate then they are Hodge-equivalent. More generally, say that $G$ and $H$ are almost conjugate if there is a bijection $\sigma: G \rightarrow H$ such that $g$ and $\sigma(g)$ are conjugate. This is the same as requiring that $g$ and $\sigma(g)$ have the same eigenvalues, so that they make the same contribution to the Hodge series. Thus almost conjugate groups are Hodge-equivalent.

It has long been known how to find non-conjugate pairs $(G, H)$ that are almost conjugate, and hence Hodge-equivalent: The 1-isospectral pairs found by Ikeda in [6] fit this bill (cf. Gilkey [4 and Ikeda [7, p. 395]). Here we are interested in pairs (and specifically, real pairs) that are Hodge-equivalent without being almost conjugate. The first such examples were given in [9] by Lauret, Miatello, and Rossetti (henceforth 'LMR'). They exhibited a multitude of examples arising already among cyclic groups. For cyclic groups, conjugacy is the same as almost-conjugacy, so their examples can be briefly described as being Hodge-equivalent without being conjugate. Our goal here is to explain, simplify, and extend their findings.

\section{ISOSPECTRALITY}

We discuss here the connection to spectral theory, which is what motivated LMR to construct their examples. This is meant for background only: In the approach 
taken here, spectral theory plays no role. In this section we restrict to real groups, which we may assume to be orthogonal.

A finite real group $G \subset O_{n}$ is classified up to conjugacy by the isometry type of the quotient orbifold $Q_{G}=G \backslash S^{n-1}$, where $S^{n-1}$ is the $(n-1)$-dimensional sphere realized as the unit sphere in $\mathbf{R}^{n}$. According to Ikeda [7], $G$ and $H$ are Hodge-equivalent just if the quotients $Q_{G}$ and $Q_{H}$ are isospectral for the Hodge Laplacian on $p$-forms for $p=0, \ldots, n-1$. According to Pesce [11], $Q_{G}$ and $Q_{H}$ are strongly isospectral (isospectral for all natural operators of a certain kind) just if $G$ and $H$ are almost conjugate. Using this dictionary, looking for Hodge-equivalent groups that are not almost conjugate is the same as looking for Hodge-isospectral orbifolds that are not strongly isospectral. This is why LMR were interested in this question.

Ikeda, Pesce, and LMR restricted their investigations to the case of groups whose action on $S_{n-1}$ is fixed-point free (no $g \neq 1$ has 1 as an eigenvalue). In this case $Q_{G}$ is a manifold, called a spherical space form. For $n$ odd (i.e. $n-1$ even) we have only the sphere $\left\{I_{n}\right\} \backslash S^{n-1}$ and projective space $\left\{ \pm I_{n}\right\} \backslash S^{n-1}$. So the restriction to fixed-point free actions effectively limits us to the case of even $n$.

When $G$ is cyclic, as in the LMR examples, $Q_{G}$ is a lens space. As observed above, for cyclic groups almost-conjugacy is the same as conjugacy, which is the same as isometry of the corresponding lens space. So we can briefly describe the LMR examples as Hodge-isospectral lens spaces that are not isometric, hence not almost conjugate, hence not strongly isospectral.

This ends our discussion of isospectrality. The rest is algebra.

\section{CyClic GROUPS}

For any $q$ and $s=\left(s_{1}, \ldots, s_{n}\right)$, write

$$
\omega_{q}^{s}=\left(\omega_{q}^{s_{1}}, \ldots, \omega_{q}^{s_{n}}\right) .
$$

Consider the finite cyclic group

$$
L(q, s)=\left\langle\operatorname{diag}\left(\omega_{q}^{s}\right)\right\rangle=\left\{\operatorname{diag}\left(\omega_{q}^{k s}\right): k \in \mathbf{Z}_{q}\right\} .
$$

This group has order $q$ if $\operatorname{gcd}\left(q, s_{1}, \ldots, s_{n}\right)=1$. Up to conjugacy, any finite cyclic subgroup of $U_{n}$ can be written in this way.

The cyclic group $L(q, s)$ doesn't change (up to conjugacy) when you rearrange the entries of $s$, or multiply them all by an element of the multiplicative group $\mathbf{Z}_{q}^{\star}$. Conversely, the groups $L(q, s)$ and $L\left(q, s^{\prime}\right)$ are conjugate just if, when viewed as multisets $\bmod q, s^{\prime}$ can be obtained from $s$ by multiplying by an invertible element.

Now take $n=2 m$, and let $\rho: G L_{m}(\mathbf{C}) \mapsto G L_{2 m}(\mathbf{R})$ be the standard embedding, so that $\rho\left(\omega_{q}^{s}\right)$ is the diagonal sum of the 2-by-2 matrices

$$
\begin{aligned}
\rho\left(\left(\left(\omega_{q}^{s_{i}}\right)\right)\right) & =\exp \left(\tau \frac{s_{i}}{q}((0,-1),(1,0))\right) \\
& =\left(\left(\cos \left(\tau s_{i} / q\right),-\sin \left(\tau s_{i} / q\right)\right),\left(\sin \left(\tau s_{i} / q\right), \cos \left(\tau s_{i} / q\right)\right)\right) .
\end{aligned}
$$

Up to conjugacy in $U_{n}$,

$$
\rho(L(q, s)) \equiv L\left(q, s^{ \pm}\right)
$$


where

$$
s^{ \pm}=\left(s_{1},-s_{1}, \ldots, s_{m},-s_{m}\right) .
$$

Let us write

$$
L^{ \pm}(q, s)=L\left(q, s^{ \pm}\right) \equiv \rho(L(q, s))
$$

For the Hodge series we have

$$
\begin{aligned}
\Lambda_{L^{ \pm}(q, s)} & =\frac{1}{q} \sum_{k} \prod_{i} \frac{\left(1+y \omega_{q}^{k s_{i}}\right)\left(1+y \omega_{q}^{-k s_{i}}\right)}{\left(1-x \omega_{q}^{k s_{i}}\right)\left(1-x \omega_{q}^{-k s_{i}}\right)} \\
& =\frac{1}{q} \sum_{k} \prod_{i} \frac{1+2 \cos \left(\tau k s_{i} / q\right) y+y^{2}}{1-2 \cos \left(\tau k s_{i} / q\right) x+x^{2}} .
\end{aligned}
$$

\section{The LMR CONSTRUCTION}

The LMR examples involve cyclic subgroups of $O_{2 m}$ of the form

$$
\rho\left(L\left(r^{2} t, r t a+1\right)\right) \equiv L^{ \pm}\left(r^{2} t, r t a+1\right),
$$

where $r>2, t \geq 1, a=\left(a_{1}, \ldots, a_{m}\right) \in \mathbf{Z}^{m}$. Since we prefer to keep our matrices diagonal we'll define

$$
\begin{aligned}
\operatorname{LMR}(r, t, a) & =L^{ \pm}\left(r^{2} t, r t a+1\right) \\
& =L\left(r^{2} t,\left(r t a_{1}+1,-r t a_{1}-1, \ldots, r t a_{m}+1,-r t a_{m}-1\right)\right) .
\end{aligned}
$$

Note. You may wish to mentally set $t=1$ : All evidence indicates that what works for $t=1$ works in general, and in particular the criterion in Theorem 1 below does not involve $t$.

As we will be seeing, what's special about the LMR construction is the following fact:

$$
(r t c+1)(r t d+1) \equiv_{r^{2} t} r t(c+d)+1 .
$$

Thus the multiplicative subgroup $\left\{r t c+1: c \in \mathbf{Z}_{r}\right\} \subset \mathbf{Z}_{r^{2} t}^{\star}$ is cyclic of order $r$, generated by $r t+1$; the map $r t c+1 \mapsto c$ takes the logarithm of $r t c+1$ base $r t+1$, and gives an isomorphism to the additive group $\mathbf{Z}_{r}$.

As a first consequence of this, notice that we can add a constant $c$ to the entries of $a$ without changing the conjugacy class:

$$
\operatorname{LMR}(r, t, a) \equiv \operatorname{LMR}(r, t, a+c) .
$$

(We use the convention that the sum of a vector and a constant is obtained by adding the constant to each entry of the vector, so that $a+c=\left(a_{1}+c, \ldots, a_{m}+c\right)$.) In fact, in this way we account for all such coincidences: Let us write

$$
a \equiv S_{m} \times \mathbf{z}_{r} a^{\prime}
$$

if for some $c, a+c$ and $a^{\prime}$ are the same as multisets $\bmod r$. Then $\operatorname{LMR}(r, t, a) \equiv$ $\operatorname{LMR}\left(r, t, a^{\prime}\right)$ just if $a \equiv_{S_{m} \times \mathbf{z}_{r}} a^{\prime}$.

All the LMR pairs are (conjugate to) pairs of the special form

$$
(\operatorname{LMR}(r, t, a), \operatorname{LMR}(r, t,-a)) .
$$

Not all such pairs are Hodge-equivalent, however. 


\section{TheOrem}

In this section we formulate a criterion for Hodge-equivalence of the LMR pair $(\operatorname{LMR}(r, t, a), \operatorname{LMR}(r, t,-a))$. While this criterion has not been shown to be necessary, it holds in all the cases (thousands and thousands!) where the LMR construction has been found to succeed.

Definition 1. Say that $a=\left(a_{1}, \ldots, a_{m}\right)$ is:

- univalent $\bmod r$ if its entries are distinct $\bmod r$;

- reversible $\bmod r$ if $a \equiv S_{m} \times \mathbf{Z}_{r}-a$;

- good $\bmod r$ if it is univalent or reversible $\bmod r$;

- hereditarily good mod $r$ if it is good mod $d$ for all d dividing $r$;

- useful mod $r$ if it is hereditarily good and irreversible mod $r$.

Any $a$ is reversible (hence good) mod 1 or 2 . So in checking hereditary goodness we need only check divisors $d>2$.

In section 9 below we will prove the following:

Theorem 1. If a is hereditarily good mod $r$ then for any $t$,

$$
\operatorname{LMR}(r, t, a) \equiv_{\Lambda} \operatorname{LMR}(r, t,-a) .
$$

If $a$ is reversible mod $r$ then $a$ is hereditarily good $\bmod r$, but in this case $\operatorname{LMR}(r, t, a)$ and $\operatorname{LMR}(r, t,-a)$ are conjugate. So this result tells us something useful only if $a$ is hereditarily good without being reversible, which is our definition of 'useful'.

\section{EXAMPLES}

$(0,1,3)$ is:

- univalent $\bmod 4,5,6, \ldots$;

- reversible $\bmod 1,2,4,5$;

- good mod any $r \neq 3$;

- hereditarily good mod any $r$ not divisible by 3 ;

- useful mod any $r \geq 7$ not divisible by 3 .

Putting $r=7,8,10, t=1$, we get Hodge-equivalent but non-conjugate pairs of orders 49,64,100; putting $r=7, t=2$ we get a pair of order 98 .

$(0,1,4)$ is:

- univalent $\bmod 5,6,7, \ldots$;

- reversible $\bmod 1,2,5,7$;

- $\operatorname{good} \bmod$ any $r \neq 3,4$;

- hereditarily good mod any $r$ not divisible by 3 or 4 ;

- useful mod any $r \geq 10$ not divisible by 3 or 4 .

Putting $r=10, t=1$ gives a pair of order 100. Together with the four pairs coming from $(0,1,3)$ above, this gives us all five inequivalent pairs with $m=3, q \leq 100$ (see Table 1 of LMR [9]). 
We'll call the simplest of these pairs the 49-pair:

$$
\begin{aligned}
(\operatorname{LMR}(7,1, & (0,1,3)), \operatorname{LMR}(7,1,(0,-1,-3))) \\
& =\left(L^{ \pm}(49,(1,8,22)), L^{ \pm}(49,(1,-6,-20))\right. \\
& =(L(49,(1,-1,8,-8,22,-22)), L(49,(1,-1,-6,6,-20,20))) \\
& \equiv(L(49,(-6,6,1,-1,15,-15)), L(49,(1,-1,-6,6,-20,20))) \\
& \equiv\left(L^{ \pm}(49,(1,6,15)), L^{ \pm}(49,(1,6,20))\right) .
\end{aligned}
$$

Here at the next-to-last step we've multiplied the list $(1,-1,8,-8,22,-22)$ by -6 mod 49 so as to get the lexicographically least representation that the computer spits out in its search for Hodge-equivalent pairs.

\section{Proof}

It is easy enough to verify that the members of the 49-pair are Hodge-equivalent by explicit computation of their Hodge series. The same goes for as many other pairs as you like, but this only gets you a finite number of examples.

Using a very explicit representation theory argument, LMR proved Hodgeequivalence of the 49-pair in a way that extends to cover all pairs of the form

$$
(\operatorname{LMR}(r, t,(0,1,3)), \operatorname{LMR}(r, t,(0,-1,-3)))
$$

with $r$ not divisible by 3 . As we have seen, this infinite family is just what we get out of Theorem 1 if we take $a=(0,1,3)$. It includes 19 of the 62 examples in the list given by LMR of all pairs with $m=3$ and $q \leq 300$.

To prove Theorem 1 in its full generality, we're going to show that the two Hodge series involved are identical as rational functions of $x$ and $y$. This comes down to a bunch of manipulations with partial fraction expansions. It all starts with the following familiar identity.

\section{Lemma 1.}

$$
\prod_{i=1}^{n} \frac{1}{x-\lambda_{i}}=\sum_{i=1}^{n} \frac{1}{x-\lambda_{i}} \prod_{j \neq i} \frac{1}{\lambda_{i}-\lambda_{j}} .
$$

Proof. This follows from the theory of partial fractions.

Alternatively, combine terms on the right over the common denominator $\prod_{i}(x-$ $\left.\lambda_{i}\right)$. The numerator is

$$
\sum_{i} \prod_{j \neq i} \frac{x-\lambda_{j}}{\lambda_{i}-\lambda_{j}}
$$

This is a polynomial of degree $n-1$ which takes the value 1 for $x=\lambda_{1}, \ldots, \lambda_{n}$. These $n$ values of $x$ are distinct (thinking of the $\lambda_{i}$ 's as indeterminates), so the numerator is identically 1 .

Proof of Theorem 1. For general $q, s \in\left(\mathbf{Z}_{q}\right)^{m}$ put

$$
H_{q, s}(x, y)=\sum_{k \in \mathbf{Z}_{q}} \prod_{i} \frac{y-\omega_{q}^{k s_{i}}}{x-\omega_{q}^{k s_{i}}}
$$


so that

$$
\Lambda_{L(q, s)}(x, y)=\frac{1}{q} \frac{(-y)^{n}}{x^{n}} H_{q, s}(1 / x,-1 / y) .
$$

Separate the sum for $H_{q, s}$ into pieces according to $\operatorname{gcd}(k, q)$ by putting

$$
H_{d, s}^{\star}=\sum_{k \in \mathbf{Z}_{d}^{\star}} \prod_{i} \frac{y-\omega_{d}^{k s_{i}}}{x-\omega_{d}^{k s_{i}}}
$$

so that

$$
H_{q, s}=\sum_{d \mid q} H_{d, s}^{\star} .
$$

To prove the theorem, we must show that if $a$ is hereditarily good mod $r$ then

$$
H_{r^{2} t,(r t a+1)^{ \pm}}=H_{r^{2} t,(-r t a+1)^{ \pm}} .
$$

Our strategy will be to show that for all $d \mid r^{2} t$ we have

$$
H_{d,(r t a+1)^{ \pm}}^{\star}=H_{d,(-r t a+1)^{ \pm}}^{\star}
$$

We dispose first of the case where $(r t a+1)^{ \pm}$is not univalent $\bmod d$. This is taken care of by the assumption that $a$ is hereditarily good, but things are not quite as straight-forward as you might be expecting, because that condition deals with divisors of $r$, and here $d$ is any divisor of $r^{2} t$.

We pass over the trivial cases $d=1,2$. Mod any $d>2$, there is no overlap between $r t a+1$ and $-(r t a+1)$, so if $(r t a+1)^{ \pm}$is not univalent mod $d$ then neither is rta.

We pause for a lemma.

Lemma 2. For $d, \alpha, \beta \in \mathbf{Z}$, suppose $d \mid \alpha \beta$. Let $d^{\prime}=d / \operatorname{gcd}(d, \beta)$. Then $d^{\prime} \mid \alpha$ and

$$
\forall \gamma \in \mathbf{Z}\left(d\left|\beta \gamma \Longleftrightarrow d^{\prime}\right| \gamma\right) \text {. }
$$

Proof. Let $e=\operatorname{gcd}(d, \beta)$, so that $d^{\prime}=d / e$.

$$
d\left|\alpha \beta \Longrightarrow d^{\prime}=d / e\right| \alpha \beta / e
$$

and $\operatorname{gcd}\left(d^{\prime}, \beta / e\right)=1$ so $d^{\prime} \mid \alpha$, and for any $\gamma$

$$
d\left|\beta \gamma \Longleftrightarrow d^{\prime}\right| \beta / e \gamma \Longleftrightarrow d^{\prime} \mid \gamma
$$

(Pretty standard stuff, admittedly.)

So suppose $d \mid r^{2} t$ and $d \mid r t\left(a_{i}-a_{j}\right)$ for $i \neq j$. Putting $\alpha=r, \beta=r t, \gamma=a_{i}-a_{j}$ in the lemma we get

$$
d^{\prime}=d / \operatorname{gcd}(d, r t) \mid \alpha=r
$$

and

$$
d^{\prime} \mid \gamma=a_{i}-a_{j}
$$

This tells us that $a$ is not univalent $\bmod d^{\prime}$, but since by assumption it is good mod any divisor of $r$, it must be reversible $\bmod d^{\prime}$ :

$$
a \equiv S_{m} \times \mathbf{z}_{d^{\prime}}-a \text {. }
$$


By the lemma, this is equivalent to

$$
r t a \equiv S_{m} \times \mathbf{Z}_{d}-r t a,
$$

hence

$$
r t a+1 \equiv_{S_{m} \times \mathbf{z}_{d}}-r t a+1 .
$$

From this we get

$$
H_{d,(r t a+1)^{ \pm}}^{\star}=H_{d,(-r t a+1)^{ \pm}}^{\star}
$$

So from here on we may assume that $(r t a+1)^{ \pm}\left(\right.$and hence also $\left.(-r t a+1)^{ \pm}\right)$ is univalent $\bmod d$, with $d \mid r^{2} t$.

Returning for a moment to the case of $H_{d, s}^{\star}$ for general $d, s$, suppose $s \in\left(\mathbf{Z}_{d}^{\star}\right)^{m}$ with all the $s_{i}$ 's distinct $\bmod d$, so that the mod- $d$ quotient $s_{j} / d s_{i}$ is defined for all $i, j$, and different from 1 for $i \neq j$. With this restriction, for $k \in \mathbf{Z}_{d}^{\star}$ we have

$$
\prod_{i} \frac{y-\omega_{d}^{k s_{i}}}{x-\omega_{d}^{k s_{i}}}=\sum_{i} \frac{y-\omega_{d}^{k s_{i}}}{x-\omega_{d}^{k s_{i}}} \prod_{j \neq i} \frac{y-\omega_{d}^{k s_{j}}}{\omega_{d}^{k s_{i}}-\omega_{d}^{k s_{j}}} .
$$

So

$$
\begin{aligned}
H_{d, s}^{\star} & =\sum_{k \in \mathbf{Z}_{d}^{\star}} \sum_{i} \frac{y-\omega_{d}^{k s_{i}}}{x-\omega_{d}^{k s_{i}}} \prod_{j \neq i} \frac{y-\omega_{d}^{k s_{j}}}{\omega_{d}^{k s_{i}}-\omega_{d}^{k s_{j}}} \\
& =\sum_{l \in \mathbf{Z}_{d}^{\star}} \frac{y-\omega_{d}^{l}}{x-\omega_{d}^{l}} \sum_{i} \prod_{j \neq i} \frac{y-\omega_{d}^{l s_{j} / d} s_{i}}{\omega_{d}^{l}-\omega_{d}^{l s_{j} / d} s_{i}} \\
& =\sum_{l \in \mathbf{Z}_{d}^{\star}} Y_{d, s}\left(x, y, \omega_{d}^{l}\right)
\end{aligned}
$$

where

$$
Y_{d, s}(x, y, w)=\frac{y-w}{x-w} \sum_{i} \prod_{j \neq i} \frac{y-w^{s_{j} / d} s_{i}}{w-w^{s_{j} / d} s_{i}} .
$$

Now recall our notation

$$
s^{ \pm}=\left(s_{1},-s_{1}, \ldots, s_{m},-s_{m}\right) .
$$

Assuming the entries of $s^{ \pm}$are all invertible and distinct $\bmod d$,

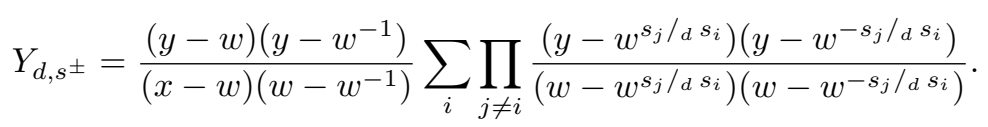

Specializing finally to the case at hand, take

$$
s=r t a+1=\left(r t a_{1}+1, \ldots, r t a_{m}+1\right)
$$

so that

$$
s^{ \pm}=\left(r t a_{1}+1,-r t a_{1}-1, \ldots, r t a_{m}+1,-r t a_{m}-1\right),
$$

and assume that these entries are all distinct $\bmod d$.

Here comes the magic: For any $d \mid r^{2} t$ we have

$$
s_{j} / d s_{i} \equiv_{d} r t\left(a_{j}-a_{i}\right)+1 \text {. }
$$


As the entries of $s^{ \pm}$are distinct $\bmod d$, putting

$$
x_{i}=w^{r t a_{i}}
$$

we have

$$
w^{s_{j} / d} s_{i}=\frac{x_{j}}{x_{i}} w
$$

and

$$
w^{-s_{j} / d s_{i}}=\frac{x_{i}}{x_{j}} w^{-1}
$$

So

$$
Y_{d,(r t a+1)^{ \pm}}=\frac{(y-w)\left(y-w^{-1}\right)}{(x-w)\left(w-w^{-1}\right)} \sum_{i} \prod_{j \neq i} \frac{\left(y-\frac{x_{j}}{x_{i}} w\right)\left(y-\frac{x_{i}}{x_{j}} w^{-1}\right)}{\left(w-\frac{x_{j}}{x_{i}} w\right)\left(w-\frac{x_{i}}{x_{j}} w^{-1}\right)} .
$$

Setting $u=y / w, v=w^{-2}$, we get

$$
Y_{r^{2} t,(r t a+1)^{ \pm}}=\frac{(y-w)\left(y-w^{-1}\right)}{(x-w)\left(w-w^{-1}\right)} F\left(\left(x_{1}, \ldots, x_{m}\right), u, v\right),
$$

where

$$
F\left(\left(x_{1}, \ldots, x_{m}\right), u, v\right)=\sum_{i=1}^{m} \prod_{j \neq i} \frac{\left(u-\frac{x_{j}}{x_{i}}\right)\left(u-\frac{x_{i}}{x_{j}} v\right)}{\left(1-\frac{x_{j}}{x_{i}}\right)\left(1-\frac{x_{i}}{x_{j}} v\right)} .
$$

Simultaneously we have

$$
Y_{r^{2} t,(-r t a+1)^{ \pm}}=\frac{(y-w)\left(y-w^{-1}\right)}{(x-w)\left(w-w^{-1}\right)} F\left(\left(1 / x_{1}, \ldots, 1 / x_{m}\right), u, v\right) .
$$

In the next section we will prove the identity

$$
F\left(\left(x_{1}, \ldots, x_{m}\right), u, v\right)=F\left(\left(1 / x_{1}, \ldots, 1 / x_{m}\right), u, v\right),
$$

from which we conclude

$$
H_{d,(r t a+1)^{ \pm}}^{\star}=H_{d,(-r t a+1)^{ \pm}}^{\star} .
$$

We have now established this last equality for every $d \mid r^{2} t$, so

$$
H_{d,(r t a+1)^{ \pm}}=H_{d,(-r t a+1)^{ \pm}} .
$$

\section{The MAIN IDENTITY}

Define the rational function

$$
\begin{aligned}
F\left(\left(x_{1}, \ldots, x_{m}\right), u, v\right) & =\sum_{i=1}^{m} \prod_{j \neq i} \frac{\left(u-\frac{x_{j}}{x_{i}}\right)\left(u-\frac{x_{i}}{x_{j}} v\right)}{\left(1-\frac{x_{j}}{x_{i}}\right)\left(1-\frac{x_{i}}{x_{j}} v\right)} \\
& =\sum_{i=1}^{m} \prod_{j \neq i} \frac{\left(x_{i} u-x_{j}\right)\left(x_{j} u-x_{i} v\right)}{\left(x_{i}-x_{j}\right)\left(x_{j}-x_{i} v\right)} .
\end{aligned}
$$


Now look at what you get by replacing the variables $x_{1}, \ldots, x_{m}$ by their reciprocals:

$$
\begin{aligned}
G\left(\left(x_{1}, \ldots, x_{m}\right), u, v\right) & =F\left(\left(1 / x_{1}, \ldots, 1 / x_{m}\right), u, v\right) \\
& =\sum_{i=1}^{m} \prod_{j \neq i} \frac{\left(u-\frac{x_{i}}{x_{j}}\right)\left(u-\frac{x_{j}}{x_{i}} v\right)}{\left(1-\frac{x_{i}}{x_{j}}\right)\left(1-\frac{x_{j}}{x_{i}} v\right)} \\
& =\sum_{i=1}^{m} \prod_{j \neq i} \frac{\left(x_{j} u-x_{i}\right)\left(x_{i} u-x_{j} v\right)}{\left(x_{j}-x_{i}\right)\left(x_{i}-x_{j} v\right)} .
\end{aligned}
$$

Proposition 1. $F=G$.

Proof. The right way to prove this identity is presumably via invariant theory. (Or maybe it's just somehow obvious?) But here we are going to prove it by considering the two sides as rational functions of $v$, expanding their individual terms in partial fractions, and seeing that the parts on the two sides agree.

The tricky case turns out to be the polynomial term, corresponding to the pole at $v=\infty$. We'll deal with that later, after we address the finite poles.

Let's look at the case $m=3$, which is sufficient to show what is going on. Each side of the identity has three terms. On the left the first term is

$$
\frac{\left(x_{1} u-x_{2}\right)\left(x_{2} u-x_{1} v\right)\left(x_{1} u-x_{3}\right)\left(x_{3} u-x_{1} v\right)}{\left(x_{1}-x_{2}\right)\left(x_{2}-x_{1} v\right)\left(x_{1}-x_{3}\right)\left(x_{3}-x_{1} v\right)} .
$$

This term is the only one on the left with non-zero residue at $v=x_{2} / x_{1}$, and its residue there is

$$
\begin{aligned}
& \frac{\left(x_{1} u-x_{2}\right)\left(x_{2} u-x_{1} x_{2} / x_{1}\right)\left(x_{1} u-x_{3}\right)\left(x_{3} u-x_{1} x_{2} / x_{1}\right)}{\left(x_{1}-x_{2}\right)\left(-x_{1}\right)\left(x_{1}-x_{3}\right)\left(x_{3}-x_{1} x_{2} / x_{1}\right)} \\
& =\frac{\left(x_{1} u-x_{2}\right) x_{2}(u-1)\left(x_{1} u-x_{3}\right)\left(x_{3} u-x_{2}\right)}{\left(x_{1}-x_{2}\right)\left(-x_{1}\right)\left(x_{1}-x_{3}\right)\left(x_{3}-x_{2}\right)} .
\end{aligned}
$$

On the right the only term with a non-zero residue at $v=x_{2} / x_{1}$ is the second term, namely

$$
\frac{\left(x_{1} u-x_{2}\right)\left(x_{2} u-x_{1} v\right)\left(x_{3} u-x_{2}\right)\left(x_{2} u-x_{3} v\right)}{\left(x_{1}-x_{2}\right)\left(x_{2}-x_{1} v\right)\left(x_{3}-x_{2}\right)\left(x_{2}-x_{3} v\right)},
$$

and the residue there is

$$
\begin{aligned}
\frac{\left(x_{1} u-x_{2}\right)\left(x_{2} u-x_{1} x_{2} / x_{1}\right)\left(x_{3} u-x_{2}\right)\left(x_{2} u-x_{3} x_{2} / x_{1}\right)}{\left(x_{1}-x_{2}\right)\left(-x_{1}\right)\left(x_{3}-x_{2}\right)\left(x_{2}-x_{3} x_{2} / x_{1}\right)} \\
=\frac{\left(x_{1} u-x_{2}\right) x_{2}(u-1)\left(x_{3} u-x_{2}\right)\left(x_{1} u-x_{3}\right)}{\left(x_{1}-x_{2}\right)\left(-x_{1}\right)\left(x_{3}-x_{2}\right)\left(x_{1}-x_{3}\right)},
\end{aligned}
$$

which is the same as we found for the left side.

In this way we see that the residues of $v$ at the finite poles all match between left and right. That leaves the pole at $v=\infty$. Taking the limit $v \rightarrow \infty$ of

$$
F\left(\left(x_{1}, \ldots, x_{m}\right), u, v\right)=\sum_{i=1}^{m} \prod_{j \neq i} \frac{\left(x_{i} u-x_{j}\right)\left(x_{j} u-x_{i} v\right)}{\left(x_{i}-x_{j}\right)\left(x_{j}-x_{i} v\right)}
$$


yields

$$
\sum_{i=1}^{m} \prod_{j \neq i} \frac{\left(x_{i} u-x_{j}\right)}{\left(x_{i}-x_{j}\right)} .
$$

In the next section, we will prove that this limit is $1+u+\cdots+u^{m-1}$, which as it is independent of $\left(x_{1}, x_{2}, x_{3}\right)$ must agree with the limit of

$$
G\left(\left(x_{1}, \ldots, x_{m}\right), u, v\right)=F\left(\left(1 / x_{1}, \ldots, 1 / x_{m}\right), u, v\right),
$$

so the residues at $v=\infty$ of the two sides of your identity match, and the proof is complete. Well, it's not a proof, exactly, since it doesn't really explain what is going on there. Call it a 'verification', which persuades us that the identity is true, at least when coupled with a symbolic computation checking the identity up through $m=4$.

\section{THE SUBSIDIARY IDENTITY}

Define the rational function

$$
f\left(\left(x_{1}, \ldots, x_{m}\right), u\right)=\sum_{i=1}^{m} \prod_{j \neq i} \frac{u-\frac{x_{j}}{x_{i}}}{1-\frac{x_{j}}{x_{i}}}=\sum_{i=1}^{m} \prod_{j \neq i} \frac{x_{i} u-x_{j}}{x_{i}-x_{j}} .
$$

\section{Proposition 2.}

$$
f\left(\left(x_{1}, \ldots, x_{m}\right), u\right)=1+u+\cdots+u^{m-1} .
$$

Proof. We use induction on $m$. The cases $m=0,1$ are trivial, and $m=2$ is so easy as not to illustrate the method. So we will look at the case $m=3$, and take that as representative. We want to show that

$$
\frac{\left(x_{1} u-x_{2}\right)\left(x_{1} u-x_{3}\right)}{\left(x_{1}-x_{2}\right)\left(x_{1}-x_{3}\right)}+\frac{\left(x_{2} u-x_{1}\right)\left(x_{2} u-x_{3}\right)}{\left(x_{2}-x_{1}\right)\left(x_{2}-x_{3}\right)}+\frac{\left(x_{3} u-x_{1}\right)\left(x_{3} u-x_{2}\right)}{\left(x_{3}-x_{1}\right)\left(x_{3}-x_{2}\right)}=1+u+u^{2} .
$$

Expand the terms on the left in partial fractions with respect to the variable $x_{3}$. The possible poles are at $x_{3}=x_{1}, x_{3}=x_{2}$, and $x_{3}=\infty$. For the coefficient of $\frac{1}{x_{3}-x_{1}}$ we get

$$
-\frac{\left(x_{1} u-x_{2}\right)\left(x_{1} u-x_{1}\right)}{x_{1}-x_{2}}+0+\frac{\left(x_{1} u-x_{1}\right)\left(x_{1} u-x_{2}\right)}{x_{1}-x_{2}} .
$$

So this coefficient vanishes (as it would have to, if our identity is to hold). Similarly for the coefficient of $\frac{1}{x_{3}-x_{2}}$. This leaves the pole at $x_{3}=\infty$. Taking the limit of the terms on the left as $x_{3} \rightarrow \infty$, we get

$$
\frac{x_{1} u-x_{2}}{x_{1}-x_{2}}+\frac{x_{2} u-x_{1}}{x_{2}-x_{1}}+u^{2}=f\left(\left(x_{1}, x_{2}\right), u\right)+u^{2}=1+u+u^{2},
$$

where in the last step we are using the induction hypothesis. 


\section{Open QUESTIONS}

(1) What is the right way to prove these two identities?

(2) LMR showed that in their construction, the full Hodge series agree just if they agree after setting $w=0$. Surely we can prove this algebraically.

(3) Is the condition in Theorem 1 for Hodge-equivalence of LMR groups necessary as well as sufficient? If true, this might not be so hard to prove. To start with, we could prove that what works for $t=1$ works for any $t$.

(4) It seems that the representation-theoretic proof of LMR might give an explicit matchup between spaces of invariant forms. Can we extract such a matchup from the algebra in the proof of Theorem 11.

(5) Not all Hodge-isospectral pairs emerge directly from the LMR construction. For example, you append a 0 to the list $r t a+1$ on both sides, or put in everything congruent to $2 \bmod r t$. It's tempting to figure out just what variations are possible. And then we could ask whether all possible pairs arise as variations of this kind.

(6) Doyle and Rossetti [3] conjectured that in spherical geometry or hyperbolic geometry, spaces that are $p$-isospectral for all $p$ are almost conjugate, and hence isospectral for all natural operators. The LMR examples show that this is false in spherical geometry, but the hyperbolic case remains open, and the intuition for this conjecture, born in the hyperbolic case and incautiously extended to the spherical case, remains more or less intact.

\section{ACKNOWLEDGEMENTS}

We thank Emilio Lauret for extensive correspondence.

\section{REFERENCES}

[1] Scott Crass. Solving the sextic by iteration: A study in complex geometry and dynamics. Experiment. Math. 8 (1999), no. 3, 209-240. MR 1724156. Preprint available: arXiv:math/9903111 [math.DS].

[2] Peter Doyle and Curt McMullen. Solving the quintic by iteration. Acta Math. 163 (1989), no. 3-4, 151-180. MR 1032073 .

[3] Peter G. Doyle and Juan Pablo Rossetti. Laplace-isospectral hyperbolic 2-orbifolds are representation-equivalent (Version 1), 2011, arXiv:1103.4372v1 [math.DG].

[4] Peter B. Gilkey. On spherical space forms with meta-cyclic fundamental group which are isospectral but not equivariant cobordant. Compositio Math. 56 (1985), no. 2, 171-200. MR 0809865

[5] Vi Hart. Pi is (still) wrong. YouTube video

[6] Akira Ikeda. On spherical space forms which are isospectral but not isometric. J. Math Soc. Japan 35 (1983), no. 3, 437-444. MR 0702768

[7] Akira Ikeda. Riemannian manifolds $p$-isospectral but not $(p+1)$-isospectral. In: Geometry of Manifolds (Matsumoto, 1988), pp. 383-417, Academic Press, 1989. MR 1040537.

[8] Felix Klein. Vorlesungen über das Ikosaeder und die Auflösung der Gleichungen vom fünften Grade. B.G. Teubner, 1884. MR 1315530. Available at the Internet Archive.

[9] Emilio A. Lauret, Roberto J. Miatello, and Juan Pablo Rossetti. Spectra of lens spaces from 1-norm spectra of congruence lattices. Int. Math. Res. Not. IMRN 2016, no. 4, 1054-1089. MR 3493442 Preprint available: arXiv:1311.7167v4 [math.DG]. 
[10] Theodor Molien. Über die Invarianten der linearen Substitutionsgruppen. Sitzungber. König. Preuss. Akad. Wiss. (J. Berl. Ber.), 52 (1897), 1152-1156.

[11] Hubert Pesce. Variétés hyperboliques et elliptiques fortement isospectrales. J. Funct. Anal. 134 (1995), no. 2, 363-391. MR 1363805

[12] Richard P. Stanley. Invariants of finite groups and their applications to combinatorics. Bull. Amer. Math. Soc. (N.S.) 1 (1979), no. 3, 475-511. MR 0526968

\section{R. DeFord}

Mathematics Department 6188 Kemeny Hall, Dartmouth College, Hanover, NH 03755, USA

daryl.r.deford.gr@dartmouth.edu

P. G. Doyle

Mathematics Department 6188 Kemeny Hall, Dartmouth College, Hanover, NH 03755, USA

doyle@math.dartmouth.edu

Received: July 10, 2017

Accepted: August 16, 2017 